\title{
Tea Polyphenols EGCG and Theaflavin Inhibit the Activity of SARS-CoV-2 3CL-Protease In Vitro
}

\author{
Minsu Jang, ${ }^{1}$ Yea-In Park, ${ }^{1}$ Yeo-Eun Cha, ${ }^{1}$ Rackhyun Park, ${ }^{1}$ Sim Namkoong, ${ }^{2}$ Jin I. Lee, \\ and Junsoo Park ${ }^{1}{ }^{1}$ \\ ${ }^{1}$ Division of Biological Science and Technology, Yonsei University, Wonju, Republic of Korea \\ ${ }^{2}$ Department of Biochemistry, Kangwon National University, Chuncheon, Republic of Korea \\ Correspondence should be addressed to Junsoo Park; junsoo@yonsei.ac.kr
}

Received 28 May 2020; Revised 14 August 2020; Accepted 9 September 2020; Published 17 September 2020

Academic Editor: Michel M. Machado

Copyright (c) 2020 Minsu Jang et al. This is an open access article distributed under the Creative Commons Attribution License, which permits unrestricted use, distribution, and reproduction in any medium, provided the original work is properly cited.

COVID-19, a global pandemic, has caused over 750,000 deaths worldwide as of August 2020. A vaccine or remedy for SARS-CoV2, the virus responsible for COVID-19, is necessary to slow down the spread and lethality of COVID-19. However, there is currently no effective treatment available against SARS-CoV-2. In this report, we demonstrated that EGCG and theaflavin, the main active ingredients of green tea and black tea, respectively, are potentially effective to inhibit SARS-CoV-2 activity. Coronaviruses require the 3CL-protease for the cleavage of its polyprotein to make individual proteins functional. EGCG and theaflavin showed inhibitory activity against the SARS-CoV-2 3CL-protease in a dose-dependent manner, and the half inhibitory concentration $\left(\mathrm{IC}_{50}\right.$ ) was $7.58 \mu \mathrm{g} / \mathrm{ml}$ for EGCG and $8.44 \mu \mathrm{g} / \mathrm{ml}$ for theaflavin. In addition, we did not observe any cytotoxicity for either EGCG or theaflavin at the concentrations tested up to $40 \mu \mathrm{g} / \mathrm{ml}$ in HEK293T cells. These results suggest that upon further study, EGCG and theaflavin can be potentially useful to treat COVID-19.

\section{Introduction}

A new coronavirus disease (COVID-19) has become the most dangerous pandemic of this century causing over 20,000,000 reported infections and over 750,000 reported deaths as of August 2020. However, a vaccine is not yet available, and effective remedies to treat COVID-19 are still under development. Therefore, the infections and deaths due to COVID-19 will continuously increase until an effective vaccine or treatment is available. For this reason, various approaches to slow down the spread of COVID-19 need to be identified and developed.

Virus-specific enzymes are most often the main targets of antiviral medicines. For example, the thymidine kinase enzyme of herpesvirus is the main target of herpesvirus therapies, and thymidine kinase inhibitors including acyclovir and ganciclovir were developed to treat herpesvirus diseases [1]. Similarly, coronavirus-specific enzymes should be potential targets to treat coronavirus diseases. The 3CLprotease is regarded as the main drug target for coronavirus diseases [2]. Cleavage of viral polyproteins by proteases is a vital step in the life cycle of coronaviruses, and several viral proteases encoded in the coronavirus RNA are required for the maturation of the viral proteins [3]. The 3CL-protease is responsible for the cleavage of viral polyproteins of coronavirus and absolutely required for replication of the virus [4-6]. In light of its importance, the 3CL-protease is a target for antivirals, and several antiviral candidates were identified using a 3CL-protease assay [7-9]. Recently, chemicals targeting the SARS-CoV-2 3CL-protease have been reported to be effective to slow down the replication of coronavirus in vivo [10].

Green tea is a popular beverage, and many reports demonstrated that green tea has health benefits including cancer prevention $[11,12]$. Epigallocatechin-3-gallate (EGCG) is the major ingredient in green tea and accounts for $50 \%$ to $80 \%$ of a brewed cup of green tea $[11,13]$. EGCG or green tea showed a wide range of antiviral activity against adenovirus, influenza virus, zika virus, herpesvirus, and hepatitis virus [14-20]. In addition, a previous report 
demonstrated that green tea has antiviral activity toward coronaviruses [21]. Theaflavin is an active ingredient of black tea, a fermented or oxidized form of green tea [22]. Theaflavin also showed antiviral activity against influenza virus, herpesvirus, rotavirus, and coronavirus [23-25].

Since previous studies suggest that the active ingredients of green tea or black tea are effective to inhibit coronavirus 3CL-protease, we examined whether EGCG and theaflavin showed inhibitory effects on SARS-CoV-2 3CL-protease. In this study, we chemically synthesized the gene encoding SARS-CoV-2 3CL-protease using the published SARS-CoV-2 genome sequence and then utilized the protease assay to evaluate the inhibitory effect of EGCG and theaflavin.

\section{Materials and Methods}

2.1. Reagents. (-)-Epigallocatechin gallate (EGCG) (E4134, purity $\geq 95 \%$ ) was purchased from Sigma-Aldrich (Saint Louis, MO). Theaflavin (No. 25129, purity $\geq 98 \%$ ) was purchased from Cayman Chemical (Ann Arbor, MI). For preparation of EGCG auto-oxidation products (EAOPs), EGCG was dissolved in $200 \mathrm{mM}$ PBS ( $\mathrm{pH} \mathrm{8.0)}$ ) at a concentration of $5 \mathrm{mg} / \mathrm{mL}$, and auto-oxidation of EGCG was carried out at $37^{\circ} \mathrm{C}$ for $0,3,6,9,12$, and 24 hours according to a previous report [26].

2.2. Preparation of SARS-CoV-2 3CL-Protease. The nucleotide sequences for SARS-CoV-2 3CL-protease were obtained from the NCBI nucleotide database (http://www.ncbi.nlm. nih.gov, Ref Seq No: NC_045512.2), and the protein-coding sequence was chemically synthesized and cloned into a pBT7 vector by Bioneer (Daejon, South Korea). The plasmid encoding His-tagged 3CL-protease was transformed into BL21 (DE3) competent cells. His-tagged SARS-CoV-2 3CLprotease expression was induced by $0.1 \mathrm{mM}$ IPTG at $18^{\circ} \mathrm{C}$ for $12 \mathrm{~h}$, and soluble 3CL-protease proteins were purified with an Ni-NTA resin (Thermo-Fisher Scientific, Rockford, IL) according to the manufacturer's protocol. The purified 3CLprotease protein was dialyzed with dialysis buffer $(137 \mathrm{mM}$ $\mathrm{NaCl}, 2.7 \mathrm{mM} \mathrm{KCl}, 10 \mathrm{mM} \mathrm{Na}_{2} \mathrm{HPO}_{4}, 1.8 \mathrm{mM} \mathrm{KH}_{2} \mathrm{PO}_{4}$, and $10 \%$ glycerol (pH 7.4)). The host strain BL21(DE3) (CP110) was purchased from Enzynomics (Daejeon, South Korea).

\subsection{Protease Assay for SARS-CoV-2 3CL-Protease. A FRET-} based protease assay was used to measure 3CL-protease activity [27]. Dabcyl-KTSAVLQSGFRKME-Edans was chemically synthesized (Anygen, Gwangju, South Korea) and used for the SARS-CoV-2 3CL-protease substrate. The $3 \mathrm{CL}$-protease activity was performed at $37^{\circ} \mathrm{C}$ using $3 \mathrm{CL}$ protein and FRET peptide in the reaction buffer $(20 \mathrm{mM}$ Tris- $\mathrm{HCl}$ ( $\mathrm{pH}$ 7.5), $200 \mathrm{mM} \mathrm{NaCl}, 5 \mathrm{mM}$ EDTA, 5 mM DTT, and $1 \%$ DMSO) for $5 \mathrm{~h}$. For the inhibition assay, the purified 3CL-protease was incubated with EGCG or theaflavin for $1 \mathrm{~h}$ before the addition of substrate. The fluorescence was measured at $528 \mathrm{~nm}$ with excitation at $360 \mathrm{~nm}$ using a Synergy HTX multimode microplate reader (Biotek, Winooski, VT). Protease activity was calculated as the difference between the activity with 3CL-protease and the activity without 3CL-protease at the indicated time.

2.4. Cell Culture and Cytotoxicity Assay. HEK293T human embryonic kidney cells were maintained in DMEM (Welgene, Seoul, Korea) containing $10 \%$ fetal bovine serum (Thermo-Fisher Scientific, Waltham, MA, USA) and antibiotic-antimycotic solution (Welgene). Cell cytotoxicity was measured using the 3-(4,5-dimethylthiazol-2-yl)-2,5diphenyltetrazolium bromide (MTT) assay. Briefly, equal number cells $\left(2 \times 10^{5}\right.$ cells/well $)$ were seeded in the wells of a 24-well plate and incubated in the presence or absence with EGCG or theaflavin. After $24 \mathrm{~h}$ incubation, MTT solution was added to a final concentration of $1 \mathrm{mg} / \mathrm{mL}$, and the mixture was incubated for an additional 3 hours. MTT was purchased from USB Corporation (Cleveland, OH, USA). HEK293T (ATCC CRL-11268) cells were obtained from ATCC (Rockville, MD, USA).

2.5. Statistical Analysis. The results of 3CL-protease activity and MTT were evaluated by a 2 -tailed Student's $t$-test using Excel software (Microsoft, Redmond, WA, USA). A $p$ value of 0.05 was considered significant. For the calculation of half inhibitory concentration $\left(\mathrm{IC}_{50}\right)$, the AAT Bioquest website program was used (https://www.aatbio.com/tools/ic50calculator-v1). The coefficient of drug interaction (CDI) was calculated to determine the drug interaction between two different drugs. CDI is defined by the following formula: $\mathrm{CDI}=\mathrm{AB} /(\mathrm{AxB})[28]$.

\section{Results}

3.1. Expression of SARS-CoV-2 3CL-Protease. Recently, the nucleotide sequence of the SARS-CoV-2 genome was published [29]. We aligned the SARS-CoV-2 3CL-protease peptide sequence with MERS 3CL-protease and SARS 3CL-protease (Figure 1(b)). Based on the amino acid sequence analysis, SARS-CoV-2 3CL-protease shares $96.08 \%$ identity with SARS 3CL-protease and $49.51 \%$ identity with MERS 3CL-protease (Figure 1(b)). Sequence alignment data indicate that SARSCoV-2 3CL-protease is more homologous to SARS 3CLprotease than MERS 3CL-protease. We used the SARS-CoV-2 3CL-protease nucleotide sequence to synthesize the gene that encodes the 3CL-protease protein. In order to conduct the SARS-CoV-2 3CL-protease assay, we expressed a His-tagged $3 \mathrm{CL}$-protease in bacteria by IPTG induction and purified the 3CL-protease by a His-tag affinity column (Figure 2(a)). SARSCoV-2 3CL-protease is readily detected in the soluble fraction, and we purified the 3CL-protease for the 3CL-protease assay (Figure 2(a)). To identify potential inhibitors of 3CL-protease activity, we used the 3CL-protease assay. As expected, the 3CLprotease assay showed higher activity as the incubation time increased up to $5 \mathrm{~h}$ (Figure 2(b)).

3.2. The Inhibitory Effect of EGCG on SARS-CoV-2 3CLProtease Activity. We examined the effect that EGCG has on the $3 \mathrm{CL}$-protease activity by testing various concentrations 
<smiles>O=C(O[C@H]1Cc2c(O)cc(O)cc2O[C@H]1c1cc(O)c(O)c(O)c1)c1cc(O)c(O)c(O)c1</smiles>

Epigallocatechin-3-gallate (EGCG)<smiles>O=c1c(O)cc(C2Oc3cc(O)cc(O)c3CC2O)cc2c(C3Oc4cc(O)cc(O)c4C[C@H]3O)cc(O)c(O)c12</smiles>

Theaflavin

(a)

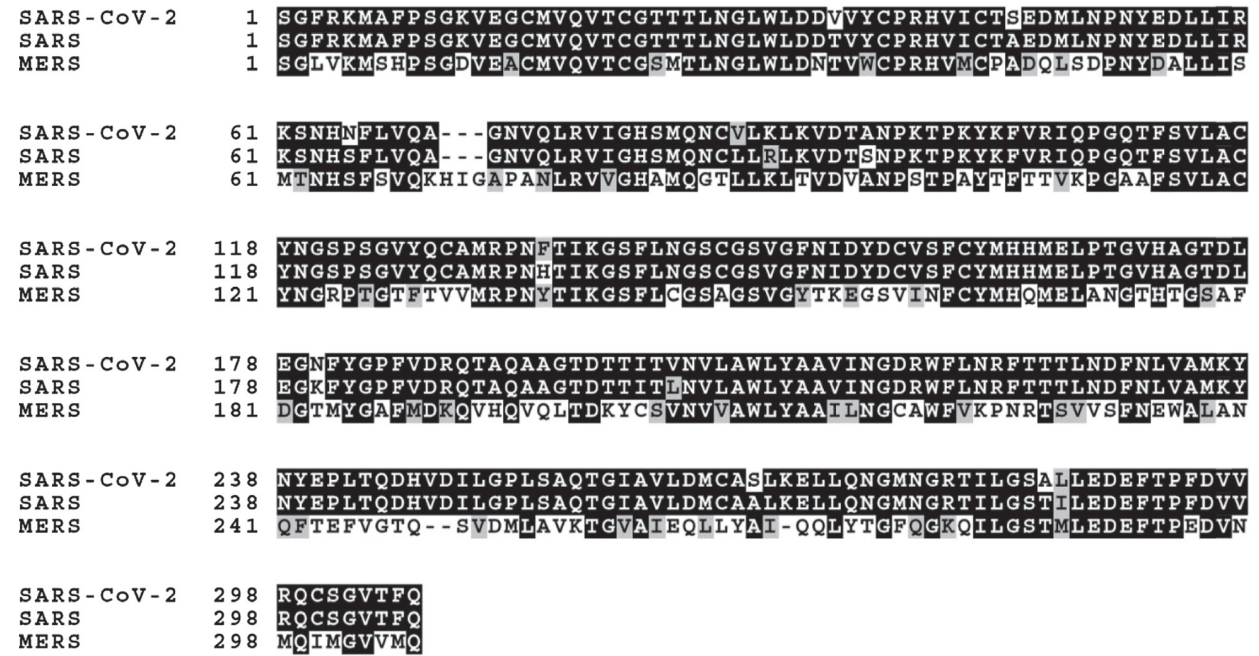

(b)

Figure 1: Amino acid sequence of SARS-CoV-2 3CL-protease. (a) Chemical structure of EGCG and theaflavin. (b) Amino acid sequence alignment of SARS-CoV-2 3CL-protease, SARS 3CL-protease, and MERS 3CL-protease.

of EGCG $(0,1,2,5,10,20$, and $40 \mu \mathrm{g} / \mathrm{ml})$. We found that EGCG significantly inhibits 3CL-protease activity in a dosedependent manner (Figure 3(a)). We calculated the half inhibitory concentration $\left(\mathrm{IC}_{50}\right)$ of EGCG using the AAT Bioquest website program and calculated an IC50 for EGCG of $7.58 \mu \mathrm{g} / \mathrm{ml}$ (Figure $3(\mathrm{~b})$ ). These results indicate that EGCG is an inhibitor of SARS-CoV-2 3CL-protease.

\subsection{The Inhibitory Effect of Theaflavin on SARS-CoV-2 3CL-} Protease Activity. Next, we examined the effect that theaflavin has on 3CL-protease activity by adding various concentrations of theaflavin $(0,1,2,5,10,20$, and $40 \mu \mathrm{g} / \mathrm{ml})$ with 3CL-protease and performing the 3CL-protease assay. The results showed that theaflavin significantly inhibits $3 \mathrm{CL}$ protease activity in a dose-dependent manner similar to EGCG (Figure 4(a)). The half inhibitory concentration $\left(\mathrm{IC}_{50}\right)$ of theaflavin was $8.44 \mu \mathrm{g} / \mathrm{ml}$, slightly higher than that of EGCG (Figure 4(b)). These results indicate that EGCG is a more effective inhibitor of SARS-CoV-2 3CL-protease activity than theaflavin.
3.4. Additive effect of EGCG and Theaflavin. Because EGCG and theaflavin both inhibit SARS-CoV-2 3CL protease, we examined whether EGCG and theaflavin together may have an additive or a synergistic inhibitory effect on 3CL-protease activity. We incubated EGCG alone, theaflavin alone, and EGCG/theaflavin and measured the inhibitory effect on 3CL-protease. Using the observed protease activity, we determined the coefficient of drug interaction (CDI) and calculated a CDI for EGCG and theaflavin of 0.93 , indicating that EGCG and theaflavin had an additive rather than a synergistic effect (Figure 5).

3.5. Inhibitory Effect of EGCG Auto-Oxidation Products (EAOPs) on SARS-CoV-2 3CL Protease. EGCG is susceptible to oxidation, and auto-oxidation of EGCG produces EGCG auto-oxidation products (EAOPs) in a time-dependent manner [26]. We examined whether EAOPs retain inhibitory activity on the $3 \mathrm{CL}$ protease. The color change indicates the production of EAOPs, and the inhibitory activity of EAOPs was not significantly changed up to $12 \mathrm{~h}$ (Figure 6). Although the inhibitory activity was decreased at $24 \mathrm{~h}$, 


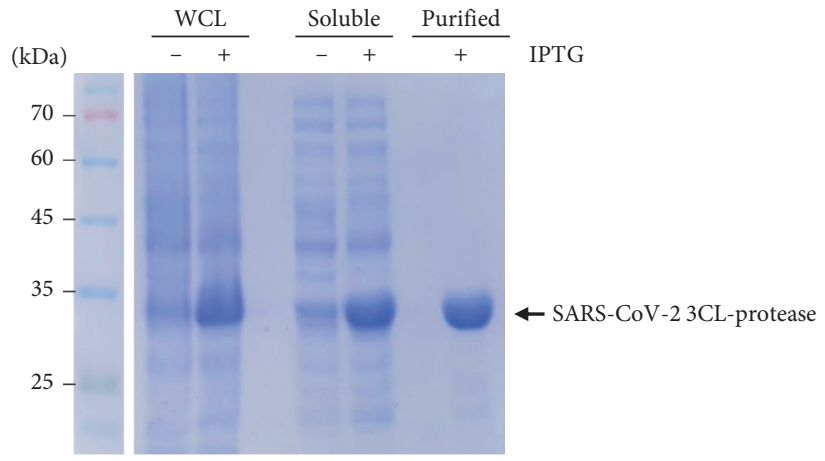

(a)

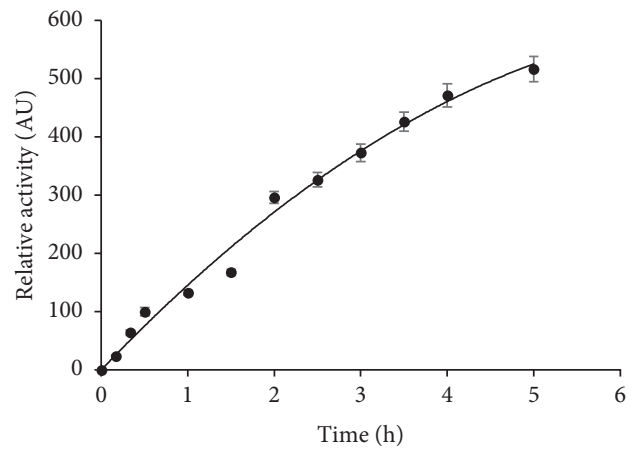

(b)

Figure 2: Expression of SARS-CoV-2 3CL-protease. (a) Purification of SARS-CoV-2 3CL-protease. Whole cell lysate (WCL), soluble fraction (soluble), and purified 3CL-protease protein (purified) in the presence or absence of IPTG were subject to SDS-PAGE. (b) Measurement of the protease activity of SARS-CoV-2 3CL-protease. AU, arbitrary unit.

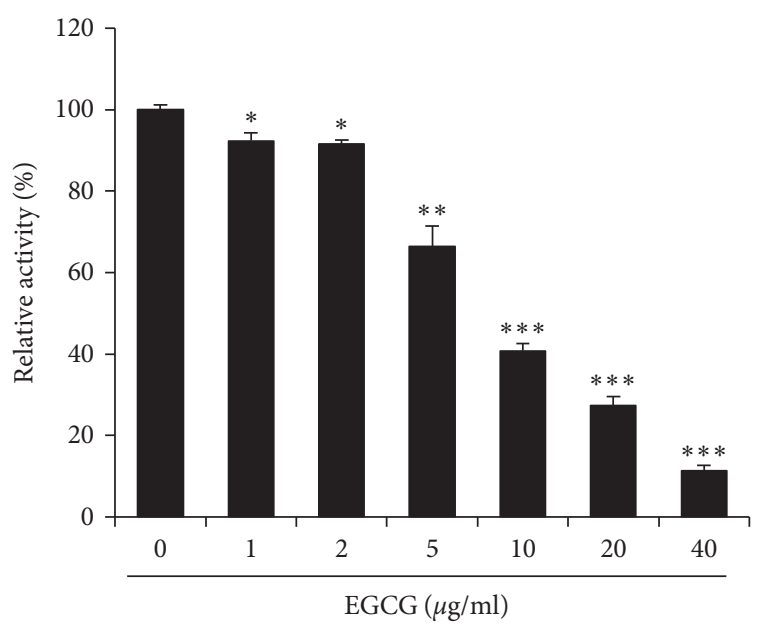

(a)

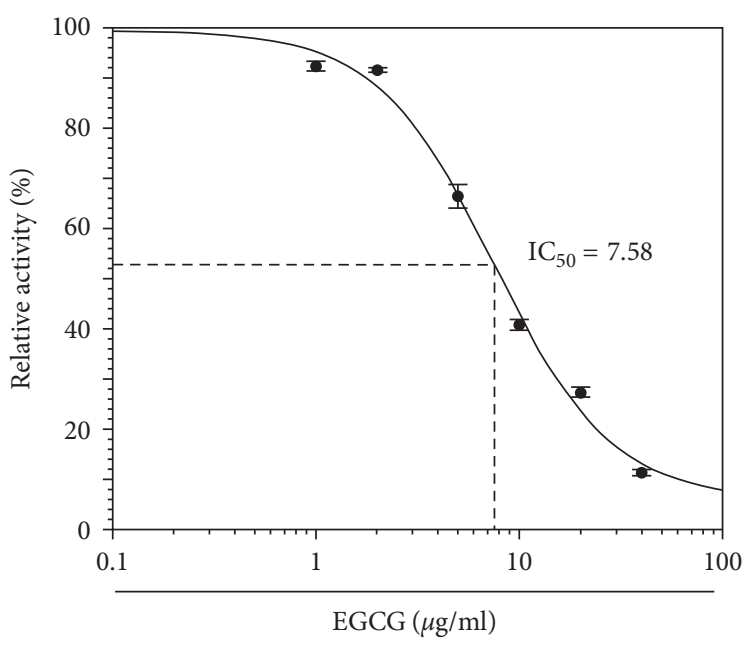

(b)

FIGURE 3: EGCG inhibits 3CL-protease in vitro. (a) Indicated concentrations of EGCG were incubated with 3CL-protease, and the 3CLprotease activity was determined. The 3CL-protease activity was performed in triplicate, and the mean and standard deviation are shown. (b) $\mathrm{IC}_{50}$ of EGCG was calculated and shown in the graph. Control vs EGCG treatment: ${ }^{*} p<0.05,{ }^{* *} p<0.01,{ }^{* * *} p<0.001$.

EAOPs still retain a significant inhibitory effect on SARSCoV-2 3CL-protease (Figure 6).

3.6. Cytotoxicity of EGCG and Theaflavin. Previous reports showed that EGCG and theaflavin showed cytotoxicity on cultured cells $[30,31]$. Therefore, we examined whether EGCG and theaflavin affected the cell viability of HEK293T cells. We incubated various concentrations of EGCG $(0,2.5,5,10,20$, and $40 \mu \mathrm{g} / \mathrm{ml})$ with HEK293T cells for $24 \mathrm{~h}$ and found that EGCG and theaflavin did not affect the cell viability significantly at any of the concentrations tested (Figure 7). These results suggest that EGCG and theaflavin can inhibit 3CL-protease without significant cellular cytotoxicity.

\section{Discussion}

The discovery for a COVID-19 treatment is urgent as COVID-19 spreads very rapidly, and infections and deaths are continuously rising all over the globe. SARS-CoV-2 3CLprotease is a promising target for COVID-19 treatments. Recently, a peptidomimetic chemical $\alpha$-ketoamide was reported to inhibit the SARS-CoV-2 3CL-protease and reduce viral replication in cell culture [10]. However, further timeconsuming testing must occur to determine the safety and efficacy of these chemicals.

In this study, we aimed to determine whether EGCG and theaflavin, the major active components of green tea and black tea, have inhibitory activity against 3CL-protease. Green tea and black tea are consumed widely and regularly 


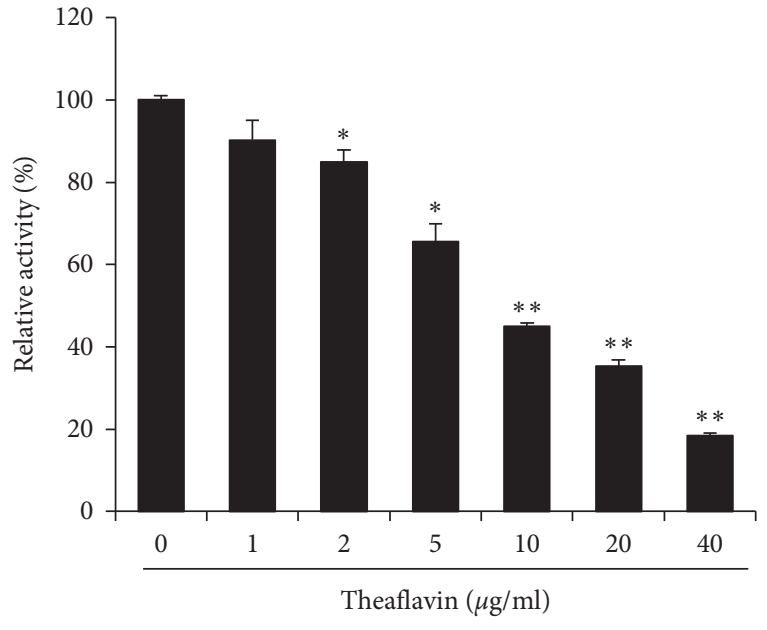

(a)

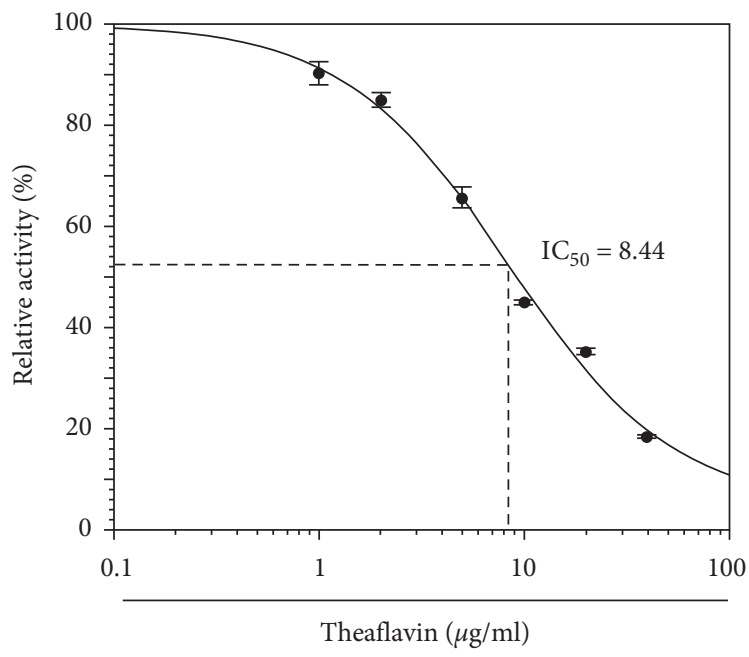

(b)

Figure 4: Theaflavin inhibits 3CL-protease in vitro. (a) Theaflavin was incubated with 3CL-protease, and the 3CL-protease activity was determined. (b) $\mathrm{IC}_{50}$ of theaflavin was calculated and shown in the graph. Control vs theaflavin treatment: ${ }^{* *} p<0.01,{ }^{* * *} p<0.001$.

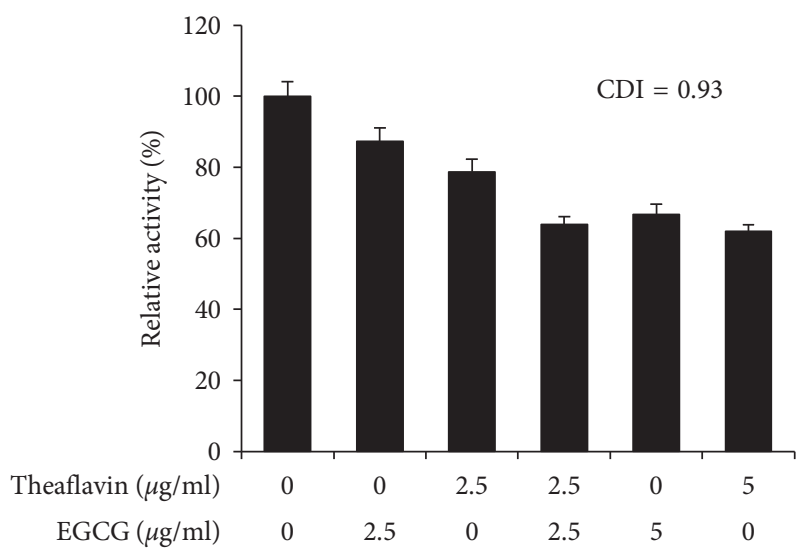

FIGURE 5: EGCG and theaflavin have an additive effect. Indicated concentration of EGCG and theaflavin were incubated with 3CLprotease, and the coefficient of drug interaction (CDI) was calculated.

throughout the world, and their health benefits have been universally lauded. We demonstrated that both EGCG and theaflavin inhibit 3CL-protease activity in a dose-dependent manner. We calculated the $\mathrm{IC}_{50}$ of EGCG and theaflavin and found $7.58 \mu \mathrm{g} / \mathrm{ml}$ for EGCG and $8.44 \mu \mathrm{g} / \mathrm{ml}$ for EGCG (Figures 3 and 4). Theaflavin inhibits SARS $3 \mathrm{CL}$-protease with an $\mathrm{IC}_{50}$ value of $7 \mu \mathrm{g} / \mathrm{ml}$, and various tea extracts were assayed against SARS coronavirus 3CLprotease, with $\mathrm{IC}_{50}$ values in the range of $25 \sim 125 \mu \mathrm{g} / \mathrm{ml}$ [21]. In addition, black tea, an oxidized form of green tea, neutralizes bovine coronavirus infectivity at an $\mathrm{EC}_{50}$ of $34.7 \mu \mathrm{g} / \mathrm{ml}$ [24]. The inhibitory activity of EGCG and theaflavin on SARS-CoV-2 3CL-protease is consistent with the inhibitory activity of SARS 3CL-protease observed in a previous study [21]. In addition, the purified single compounds (EGCG and theaflavin) show lower $\mathrm{IC}_{50}$ than tea extracts, suggesting that EGCG and
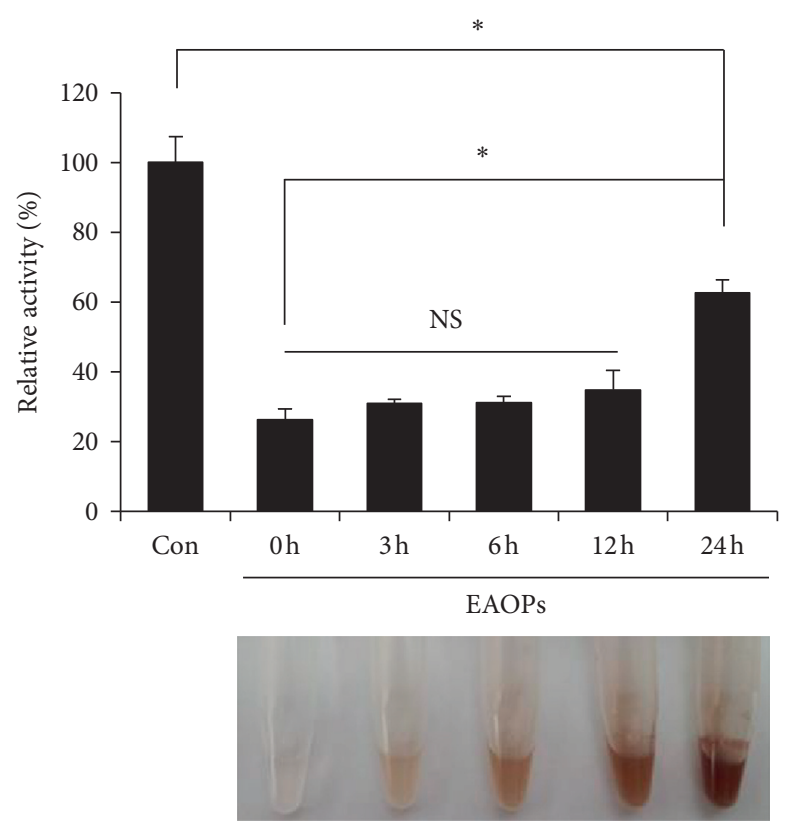

FIGURE 6: EGCG auto-oxidation products (EAOPs) show the inhibitory effect on SARS-CoV-2 3CL protease. Auto-oxidation of EGCG was carried out to produce EAOPs for the indicated hours. EAOPs $(10 \mu \mathrm{g} / \mathrm{ml})$ were incubated with $3 \mathrm{CL}$-protease, and the $3 \mathrm{CL}$ protease activity was determined (top panel). "Con", mock control. The mean and standard deviation are shown. ${ }^{*} p<0.05$; NS, not significant. Time-dependent color changes of EAOPs were shown (bottom panel).

theaflavin are the main active ingredients that inhibit 3CL-protease.

One major question is whether effective levels of either EGCG or theaflavin can be reached in the body. When we calculated the $\mathrm{IC}_{50}$ using the molar concentration of EGCG or theaflavin, the $\mathrm{IC}_{50}$ is equal to $16.5 \mu \mathrm{M}$ for EGCG and 


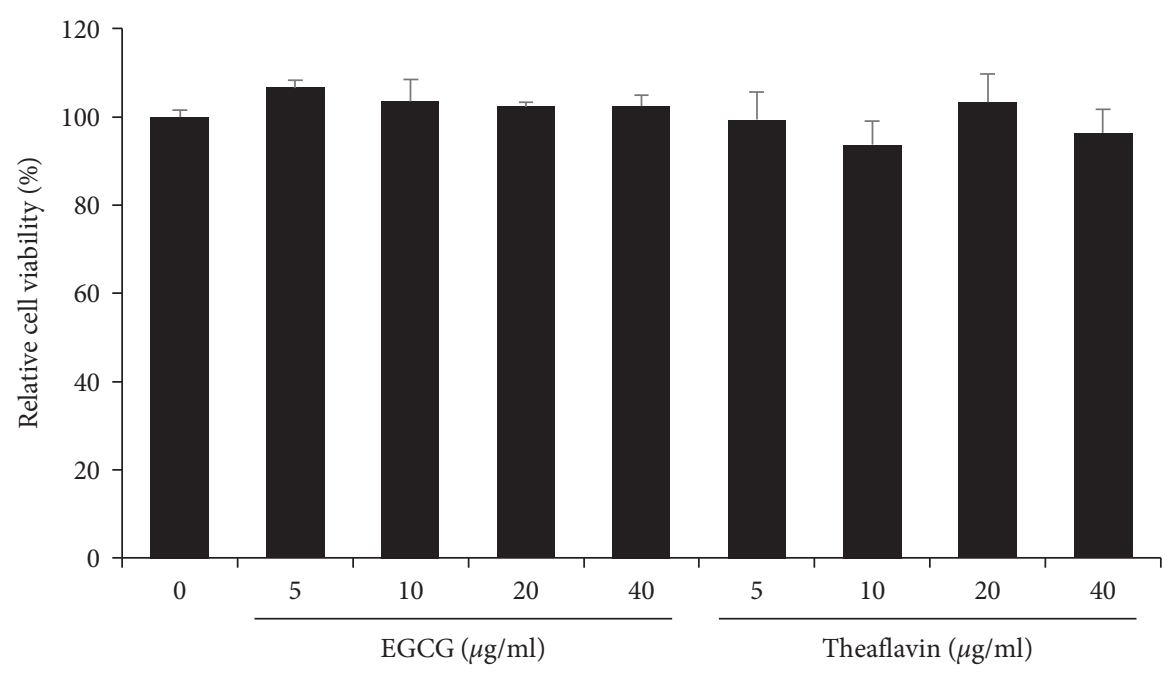

FIGURE 7: Cytotoxicity of EGCG and theaflavin. EGCG and theaflavin did not have significant cytotoxicity up to $40 \mu \mathrm{g} / \mathrm{ml}$. HEK293T cells were incubated with either EGCG or theaflavin for $24 \mathrm{~h}$, and the MTT assay was performed to evaluate the cytotoxicity.

$15.0 \mu \mathrm{M}$ for theaflavin. However, the maximum blood concentration of EGCG is less than $1 \mu \mathrm{M}$ and the maximum blood concentration of theaflavin is less than $0.1 \mu \mathrm{M}$ [32-34]. The effect of EGCG or theaflavin, therefore, on coronavirus replication in the human body may be limited on their own. However, our data also showed that EGCG and theaflavin treatment together has an additive effect (Figure 5). Green tea or black tea may provide various EGCG and theaflavin derivatives, and their additive effect may be effective to limit coronavirus replication. Moreover, EGCG can be oxidized in physiological conditions of body and converted into EAOPs. Here, we showed that EAOPs retain inhibitory activity on the 3CL-protease, suggesting that EGCG can be effective for a long time (Figure 6).

In addition, recent computational studies support that EGCG and theaflavin are inhibitors of SARS-CoV-2 3CLprotease. An in silico molecular docking study with SARSCoV-2 3CL-protease showed that EGCG and theaflavin strongly interact with 3CL-protease, indicating that EGCG and theaflavin potentially inhibit SARS-CoV-2 3CL-protease [35-37].

Here, we showed that EGCG and theaflavin, the active ingredients of green tea and black tea, are effective to inhibit the 3CL-protease in vitro. Because green tea and black tea contain a high percent of EGCG and theaflavin, it would be valuable to examine the effect of green tea and black tea on the spread of SARS-CoV-2 in vivo. In addition, further clinical trials will be required to reveal the effect of tea consumption on COVID-19 prognosis.

\section{Abbreviations}

COVID-19: 2019 novel coronavirus disease

SARS-CoV-2: Severe acute respiratory syndrome coronavirus 2

3CL-protease: 3C-like protease

EGCG: (-)-Epigallocatechin-3-gallate.

\section{Data Availability}

The original data that support the findings of this study are included in the article.

\section{Conflicts of Interest}

The authors declare that they have no conflicts of interest.

\section{Authors' Contributions}

Minsu Jang and Yea-In Park contributed equally to this work.

\section{Acknowledgments}

This study was supported by a National Research Foundation of Korea (NRF) grant funded by the Korean government (2019R1A2C1006511).

\section{References}

[1] P. Reusser, "Herpesvirus resistance to antiviral drugs: a review of the mechanisms, clinical importance and therapeutic options," Journal of Hospital Infection, vol. 33, no. 4, pp. 235248, 1996.

[2] K. Anand, J. Ziebuhr, P. Wadhwani, J. R. Mesters, and R. Hilgenfeld, "Coronavirus main proteinase (3CLpro) structure: basis for design of anti-SARS drugs," Science, vol. 300, no. 5626, pp. 1763-1767, 2003.

[3] J. Herold, A. E. Gorbalenya, V. Thiel, B. Schelle, and S. G. Siddell, "Proteolytic processing at the amino terminus of human coronavirus 229E gene 1-encoded polyproteins: identification of a papain-like proteinase and its substrate," Journal of Virology, vol. 72, no. 2, pp. 910-918, 1998.

[4] M. F. Hsu, C. J. Kuo, K. T. Chang et al., "Mechanism of the maturation process of SARS-CoV 3CL protease," Journal of Biological Chemistry, vol. 280, no. 35, pp. 31257-31266, 2005. 
[5] Y. Kim, S. R. Mandadapu, W. C. Groutas, and K. O. Chang, "Potent inhibition of feline coronaviruses with peptidyl compounds targeting coronavirus 3C-like protease," Antiviral Research, vol. 97, no. 2, pp. 161-168, 2012.

[6] A. Hegyi and J. Ziebuhr, "Conservation of substrate specificities among coronavirus main proteases," Journal of General Virology, vol. 83, no. 3, pp. 595-599, 2002.

[7] L. R. Chen, Y. C. Wang, Y. W. Lin et al., "Synthesis and evaluation of isatin derivatives as effective SARS coronavirus 3CL protease inhibitors," Bioorganic \& Medicinal Chemistry Letters, vol. 15, no. 12, pp. 3058-3062, 2005.

[8] J. T. Hsu, C. J. Kuo, H. P. Hsieh et al., "Evaluation of metalconjugated compounds as inhibitors of 3CL protease of SARSCoV," FEBS Letters, vol. 574, no. 1-3, pp. 116-120, 2004.

[9] S. Yang, S. J. Chen, M. F. Hsu et al., "Synthesis, crystal structure, structure-activity relationships, and antiviral activity of a potent SARS coronavirus 3CL protease inhibitor," Journal of Medicinal Chemistry, vol. 49, no. 16, pp. 4971-4980, 2006.

[10] L. Zhang, D. Lin, X. Sun et al., "Crystal structure of SARSCoV-2 main protease provides a basis for design of improved alpha-ketoamide inhibitors," Science, vol. 49, 2020.

[11] B. N. Singh, S. Shankar, and R. K. Srivastava, "Green tea catechin, epigallocatechin-3-gallate (EGCG): mechanisms, perspectives and clinical applications," Biochemical Pharmacology, vol. 82, no. 12, pp. 1807-1821, 2011.

[12] J. D. Lambert and R. J. Elias, "The antioxidant and pro-oxidant activities of green tea polyphenols: a role in cancer prevention," Archives of Biochemistry and Biophysics, vol. 501, no. 1, pp. 65-72, 2010.

[13] H. N. Graham, "Green tea composition, consumption, and polyphenol chemistry," Preventive Medicine, vol. 21, no. 3, pp. 334-350, 1992.

[14] J. M. Weber, A. Ruzindana-Umunyana, L. Imbeault, and S. Sircar, "Inhibition of adenovirus infection and adenain by green tea catechins," Antiviral Research, vol. 58, no. 2, pp. 167-173, 2003.

[15] S. Ciesek, T. von Hahn, C. C. Colpitts et al., "The green tea polyphenol, epigallocatechin-3-gallate, inhibits hepatitis C virus entry," Hepatology, vol. 54, no. 6, pp. 1947-1955, 2011.

[16] K. Kaihatsu, M. Yamabe, and Y. Ebara, "Antiviral mMechanism of aAction of eEpigallocatechin-3-O-gallate and iIts fFatty aAcid eEsters," Molecules, vol. 2310 pages, 2018.

[17] J. M. Song, K. H. Lee, and B. L. Seong, "Antiviral effect of catechins in green tea on influenza virus," Antiviral Research, vol. 68, no. 2, pp. 66-74, 2005.

[18] B. M. Carneiro, M. N. Batista, A. C. S. Braga, M. L. Nogueira, and P. Rahal, "The green tea molecule EGCG inhibits Zika virus entry," Virology, vol. 496, pp. 215-218, 2016.

[19] J. P. Tsai, K. C. Tseng, M. N. Lin, and C. C. Su, "A high seroprevalence of human herpesvirus type 8 already present in patients with chronic hepatitis before the development of cirrhosis," Pathology, vol. 51, no. 1, pp. 86-90, 2019.

[20] J. Steinmann, J. Buer, T. Pietschmann, and E. Steinmann, "Anti-infective properties of epigallocatechin-3-gallate (EGCG), a component of green tea," British Journal of Pharmacology, vol. 168, no. 5, pp. 1059-1073, 2020.

[21] C. N. Chen, C. P. Lin, K. K. Huang et al., "Inhibition of SARSCoV 3C-like pProtease aActivity by tTheaflavin-3,3'-digallate (TF3)," Evidence-Based Complementary and Alternative Medicine, vol. 2, no. 2, pp. 209-215, 2005.

[22] L. K. Leung, Y. Su, R. Chen, Z. Zhang, Y. Huang, and Z. Y. Chen, "Theaflavins in black tea and catechins in green tea are equally effective antioxidants," Journal of Nutrition, vol. 131, no. 9, pp. 2248-2251, 2001.
[23] M. Zu, F. Yang, W. Zhou, A. Liu, G. Du, and L. Zheng, "In vitro anti-influenza virus and anti-inflammatory activities of theaflavin derivatives," Antiviral Research, vol. 94, no. 3, pp. 217-224, 2012.

[24] K. J. Clark, P. G. Grant, A. B. Sarr et al., "An in vitro study of theaflavins extracted from black tea to neutralize bovine rotavirus and bovine coronavirus infections," Veterinary Microbiology, vol. 63, no. 2-4, pp. 147-157, 1998.

[25] C. E. Isaacs and W. Xu, "Theaflavin-3,3'-digallate and lactic acid combinations reduce herpes simplex virus infectivity," Antimicrobial Agents and Chemotherapy, vol. 57, no. 8, pp. 3806-3814, 2013.

[26] Y. Wei, P. Chen, T. Ling et al., "Certain (-)-epigallocatechin3-gallate (EGCG) auto-oxidation products (EAOPs) retain the cytotoxic activities of EGCG," Food Chemistry, vol. 204, pp. 218-226, 2016.

[27] S. Jo, S. Kim, D. H. Shin, and M. S. Kim, "Inhibition of SARSCoV 3CL protease by flavonoids," Journal of Enzyme Inhibition and Medicinal Chemistry, vol. 35, no. 1, pp. 145-151, 2020.

[28] Y. Zhao, J. L. Gao, J. W. Ji et al., "Cytotoxicity enhancement in MDA-MB-231 cells by the combination treatment of tetrahydropalmatine and berberine derived from Corydalis yanhusuo W. T. Wang," Journal of Intercultural Ethnopharmacology, vol. 3, no. 2, pp. 68-72, 2014.

[29] N. Zhu, D. Zhang, W. Wang et al., "A nNovel cCoronavirus from pPatients with pPneumonia in China," The New England Journal of Medicine, vol. 382, no. 8, pp. 727-733, 2019.

[30] W. G. Chung, C. L. Miranda, and C. S. Maier, "Epigallocatechin gallate (EGCG) potentiates the cytotoxicity of rotenone in neuroblastoma SH-SY5Y cells," Brain Research, vol. 1176, pp. 133-142, 2007.

[31] H. Babich, S. M. Pinsky, E. T. Muskin, and H. L. Zuckerbraun, "In vitro cytotoxicity of a theaflavin mixture from black tea to malignant, immortalized, and normal cells from the human oral cavity," Toxicology In Vitro, vol. 20, no. 5, pp. 677-688, 2006.

[32] K. Nakagawa and T. Miyazawa, "Absorption and distribution of tea catechin, (-)-epigallocatechin-3-gallate, in the rat," The Journal of Nutritional Science and Vitaminology, vol. 43, no. 6, pp. 679-684, 1997.

[33] L. C. Lin, L. C. Hung, and T. H. Tsai, "Determination of (-)-epigallocatechin gallate in rat blood by microdialysis coupled with liquid chromatography," Journal of Chromatography A, vol. 1032, no. 1-2, pp. 125-128, 2004.

[34] T. P. Mulder, C. J. van Platerink, P. J. Wijnand Schuyl, and J. M. van Amelsvoort, "Analysis of theaflavins in biological fluids using liquid chromatography-electrospray mass spectrometry," Journal of Chromatography B: Biomedical Sciences, vol. 760, no. 2, pp. 271-279, 2001.

[35] R. Ghosh, A. Chakraborty, A. Biswas, and S. Chowdhuri, "Evaluation of green tea polyphenols as novel corona virus (SARS CoV-2) main protease (Mpro) inhibitors-an in silico docking and molecular dynamics simulation study," Journal of Biomolecular Structure and Dynamics, vol. 760, pp. 1-13, 2020.

[36] K. A. Peele, P. Chandrasai, T. Srihansa et al., "Molecular docking and dynamic simulations for antiviral compounds against SARS-CoV-2: aA computational study," Inform Med Unlocked, vol. 76, 2020.

[37] V. K. Bhardwaj, R. Singh, J. Sharma, V. Rajendran, R. Purohit, and S. Kumar, "Identification of bioactive molecules from tea plant as SARS-CoV-2 main protease inhibitors," Journal of Biomolecular Structure and Dynamics, vol. 60, pp. 1-10, 2020. 